\title{
Correlates Between Host and Viral Transcriptional Program Associated with Different Oncolytic Vaccinia Virus Isolates
}

\author{
Jennifer Reinboth, ${ }^{1,2,3}$ Maria L. Ascierto,,4 Nanhai G. Chen,, ${ }^{1,5}$ Qian Zhang, ${ }^{1,5}$ Yong A. Yu, ${ }^{1,5}$ \\ Richard J. Aguilar, Rafael Carretero, ${ }^{2,6}$ Andrea Worschech, ${ }^{7}$ Yingdong Zhao, ${ }^{8}$ Ena Wang, \\ Francesco M. Marincola, and Aladar A. Szalay ${ }^{1,3,5,9}$
}

\begin{abstract}
Vaccinia virus (VACV) has emerged as an attractive tool in oncolytic virotherapy. VACV replication efficiency plays a crucial role in the therapeutic outcome. However, little is known about the influence of host factors on viral replication efficiency and permissiveness of a host cell line to infection and oncolysis. In this study, replication of the attenuated VACV GLV-1h68 strain and three wild-type VACV isolates was determined in two autologous human melanoma cell lines (888-MEL and 1936-MEL). Host gene expression and viral gene expression in infected cells were evaluated via respective expression array platforms.

Microarray analyses followed by sequential statistical approaches characterized human genes that change specifically due to virus infection. Viral gene transcription correlated with viral replication in a time-dependent manner. A set of human genes revealed strong correlations with the respective viral gene expression. Finally we identified a set of human genes with possible predictive value for viral replication in an independent dataset.

The results demonstrate a probable correlation between viral replication, early gene expression, and the respective host response, and thus a possible involvement of human host factors in viral early replication. The characterization of human target genes that influence viral replication could help answer the question of host cell permissiveness to oncolytic virotherapy and provide important information for the development of novel recombinant vaccinia viruses with improved features to enhance replication rate and hence trigger therapeutic outcome.
\end{abstract}

\section{Introduction}

D ESPITE EXTENSIVE RESEARCH, cancer, particularly at advanced stages, remains untreatable. For this reason, novel therapeutic strategies are needed. A promising approach is the use of oncolytic viruses. Oncolytic virotherapy exploits the natural or artificial tumor tropism of certain viruses to specifically target, replicate in, and lyse tumor cells without harming healthy somatic cells (Zeh and Bartlett, 2002; Guo et al., 2008).
Vaccinia virus (VACV), which belongs to the poxvirus family, has a large double-stranded DNA genome, enabling insertion of up to $25 \mathrm{~kb}$ of foreign DNA (Smith and Moss, 1983) without loss of infectivity, and because of its high infection efficiency, VACV has emerged as an attractive tool for gene delivery (Falkner and Moss, 1990). The recombinant VACV GLV-1h68, derived from vaccinia virus Lister strain (LIVP), contains three marker gene insertions that not only attenuate the virus but also serve diagnostic purposes. The expression cassettes encoding Renilla luciferase-green

\footnotetext{
${ }^{1}$ Genelux Corporation, San Diego Science Center, San Diego, CA 92109.

${ }^{2}$ Infectious Disease and Immunogenetics Section, Department of Transfusion Medicine, CC, and trans-NIH Center for Human Immunology, National Institutes of Health, Bethesda, MD 20892.

${ }^{3}$ Department of Biochemistry, University of Wuerzburg, Wuerzburg 97074, Germany.

${ }^{4}$ Department of Health Sciences and Center of Excellence for Biomedical Research, University of Genoa, Genoa 16132, Italy.

${ }_{5}^{5}$ Department of Radiation Oncology, Rebecca and John Moores Comprehensive Cancer Center, University of California, San Diego, CA 92093.

${ }^{6}$ Dept Bioquímica, Biología Molecular e Inmunologia, University of Granada, Granada 18071, Spain.

${ }^{7}$ Department of Internal Medicine II, University of Wuerzburg, Wuerzburg 97080, Germany.

${ }^{8}$ Biometric Research Branch, Division of Cancer Treatment and Diagnosis, National Cancer Institute, National Institutes of Health, Bethesda, MD 20852.

${ }^{9}$ Rudolf Virchow Center for Experimental Biomedicine and Institute for Molecular Infection Biology, University of Wuerzburg, Wuerzburg 97080, Germany.
} 
fluorescent protein (GFP, Aequorea) fusion, beta-galactosidase, and beta-glucuronidase were inserted into the $F 14.5 \mathrm{~L}$, $J 2 R$ (coding for thymidine kinase), and A56R (coding for hemagglutinin) loci of the parental virus genome, respectively (Zhang et al., 2007). Insertion of the three expression cassettes resulted in the attenuation of the parental virus by both inactivation of the three loci and expression of the three marker genes. It was reported previously that thymidine kinase (TK)-deleted VACV mutants depended on a thymidine triphosphate (TTP) source for viral DNA synthesis, leading to a preferential viral replication in metabolically active cells such as cancer cells with only minimal infection of normal tissues (Buller et al., 1985; Puhlmann et al., 2000; McCart et al., 2001). Further, it was published that alterations in the hemagglutinin gene resulted in a decrease in virulence in Western Reserve (WR) or New York City Board of Health (NYCBH) strains (Flexner et al., 1987; Shida et al., 1988; Lee et al., 1992). The disruption of the F14.5L locus has an attenuating effect as well (Izmailyan and Chang, 2008; Zhang et al., 2009), but function of F14.5 and why perturbation of this gene leads to attenuation is still unclear. In addition, it was found recently that removal of each of the expression cassettes from GLV-1h68 resulted in enhanced virus replication and virulence in mice. The increase in virus replication efficiency was proportionate to the strength of removed VACV promoters linked to foreign genes (Chen et al., 2011).

It has been shown that GLV-1h68 is capable of colonizing xenografts from several solid cancers and affecting their growth. Among them are breast (Zhang et al., 2007), pancreatic (Yu et al., 2009a), prostate (Gentschev et al., 2010), squamous cell (Yu et al., 2009b), anaplastic thyroid (Lin et al., 2007; Kelly et al., 2008), hepatocellular carcinoma (Gentschev et al., 2011), and malignant pleural mesothelioma (Kelly et al., 2008).

VACV, like all other members of the poxvirus family, features a unique cytoplasmic life cycle. The $200 \mathrm{kbp}$-genome encodes for more than 200 proteins involved in gene expression, DNA synthesis, virion morphogenesis, and host defense/immune evasion (Moss, 2007). VACV genes are expressed in a cascade-like manner and are therefore subdivided into three temporal classes: early, intermediate, and late. After poxvirus infection, a series of virus-induced alterations of host's cellular function occur to benefit virus replication and avert virus recognition and antiviral defense (Guerra et al., 2003). In addition, VACV infection induces an inhibition of host DNA replication, RNA transcription, and protein synthesis.

The enzymes required for early gene transcription (Broyles and Knutson, 2010) are pre-packaged in the virus core. Therefore, early transcription is completely independent from cellular factors. Intermediate and late gene expression requires more complex initiation and regulation strategies than early gene expression. Assumptions about VACV efficiency of intermediate and late transcription based on promoter element spacing and involvement of host cell factors like TBP (TATA-box binding protein) were reviewed by Broyles and Knutson (2010). It is known that the initiation of viral DNA synthesis is crucial for the beginning of intermediate gene transcription.

Five viral early gene products are essential for VACV replication: DNA polymerase (E9), processivity factor (A20), NTPase/primase (D5), uracil DNA glycosylase (D4), and protein kinase (B1). Although most of the factors involved in
VACV DNA synthesis are conserved within the poxvirus family, the replication of different VACV strains and isolates varies significantly. Further, the analysis of the NCI-60 panel of cell lines revealed that the replication efficiency of the same virus strain (GLV-1h68) is quite heterogeneous among different cancer cell lines and independent of the tissue from which the cell lines were generated (Ascierto et al., 2011). Thus, because of the lack of consistency in the behavior of different VACV isolates in infecting a given cell line and the large variability in the efficiency with which distinct VACV isolates infect different cell lines, the identification of predictors of permissiveness to VACV infection and replication remains elusive.

In this study, we focused on early events related to VACV infection to identify host genes that might influence viral replication and indicate permissiveness. One general aim was to understand the underlying kinetics of viral replication and transcription. The use of a 36k whole genome human array platform, as well as a custom-made VACV array, allowed us to analyze viral and host gene expression simultaneously. We observed a correlation between viral early replication, VACV early gene expression, and the respective host response. Further, we identified 10 human candidate genes as possible predictors for viral replication in an independent dataset.

The characterization of human target genes that influence viral replication and/or amplification could help the development of novel recombinant oncolytic VACV with enhanced replication potential and potentially better therapeutic outcome.

\section{Material and Methods}

\section{Cell lines}

The two melanoma cell lines 888-MEL and 1936-MEL were derived from sequential subcutaneous metastases in patient 888 as described previously (Wang et al., 2006; Sabatino et al., 2008). The cell lines were cultured in Roswell Park Memorial Institute medium (RPMI) supplemented with $10 \%$ fetal bovine serum (FBS), N-2-hydroxyethylpiperazine$\mathrm{N}^{\prime}$-2-ethanesulphonic acid (16mM HEPES), 1\% Ciprofloxacin, and $1 \%$ antibiotic-antimycotic solution (AA) $(100 \mathrm{U} / \mathrm{mL}$ penicillin G, $250 \mathrm{ng} / \mathrm{mL}$ amphotericin B, $100 \mu \mathrm{g} / \mathrm{mL}$ streptomycin). GI101A, a metastatic breast tumor cell line, was kindly provided by Dr. A. Aller (Rumbaugh-Goodwin Institute for Cancer Research, Inc., Plantation, Florida). HT29 colon adenocarcinoma cells were purchased from American Type Culture Collection (ATCC, Manassas, VA). Both cell lines were cultured in RPMI medium with the following supplements, GI101A cells: 20\% FBS, $4.5 \mathrm{~g} / \mathrm{L}$ glucose, 10mM HEPES, $1 \mathrm{mM}$ sodium pyruvate, $1 \% \mathrm{AA}, 4 \mathrm{ng} / \mathrm{mL} \beta$-estradiol, $5 \mathrm{ng} / \mathrm{mL}$ progesterone; and HT-29 cells: 10\% FBS and 1\% AA. African green monkey kidney fibroblasts (CV-1) were cultured in Dulbecco's modified Eagle's medium (DMEM) supplemented with 10\% FBS and 1\% AA. CV-1 cells were obtained from the ATCC. All cells were maintained at $37^{\circ} \mathrm{C}$ under $5 \% \mathrm{CO}_{2}$.

\section{Viral constructs and infections}

The construction of replication competent, recombinant VACV GLV-1h68 was described previously (Zhang et al., 2007). In brief, three expression cassettes (coding for Renilla 
luciferase-green fluorescent protein (Aequorea) fusion, $\beta$ galactosidase, and $\beta$-glucoronidase cDNA) were inserted into the $F 14.5 \mathrm{~L}, J 2 \mathrm{R}$ and $A 56 \mathrm{R}$ loci, respectively, of the wild type (wt) LIVP genome. Different wt clones were picked from the mixed population of the LIVP strain. Three isolates that showed natural attenuations due to point mutations and deletions were picked for further analysis, namely LIVP 1.1.1, LIVP 5.1.1, and LIVP 6.1.1. The generation of the recombinant VACV strains GLV-1h70, GLV-1h71, GLV-1h72, GLV-1h73, and GLV-1h74 was described previously (Chen et al., 2011).

\section{Viral replication assay}

888-MEL and 1936-MEL cells were infected with each virus in six-well plates at a multiplicity of infection (MOI) of 0.01. Cells were harvested at 2, 6, 10, 24, and 48 hours post infection (hpi). Viral particles were released by applying three freeze \& thaw cycles. Viral titers were determined by standard plaque assays on CV-1 cell monolayers. Each cell lysate was titered in duplicate and the determined titer expressed in pfu/ $10^{6}$ cells.

\section{Total RNA isolation and amplification}

Total RNA (tRNA) from tissue cultures was isolated with the Qiagen miRNeasy Mini kit, and quality and quantity of the RNA were determined with the Agilent Bioanalyzer 2000. For expression analyses on a human 36k platform, the tRNA was amplified into antisense RNA (aRNA) as previously described (Wang et al., 2000). As a reference for human arrays, peripheral blood mononuclear cells (PBMCs) from four normal donors were pooled. For viral expression analysis on a customized Affymetrix platform, tRNA was amplified into sense RNA by using the GeneChip ${ }^{\circledR}$ One-Cycle Target Labeling and Control kit for HT29 and GI101A. Since this product has been discontinued, aRNA of both melanoma cell lines was generated by the GeneChip ${ }^{\circledR}$ 3' IVT Express Kit according to the manufacturer's instructions.

\section{Microarray performance}

Array quality was documented as previously described (Jin et al., 2004). For hybridization of the 36k human array, a two-color system was applied. Both reference and sample aRNA were labeled directly using ULS ${ }^{\mathrm{TM}}$ (Universal Linkage System) aRNA Fluorescent Labeling kit with Cy3 and Cy5, respectively. Samples and references were cohybridized to the array slides (Worschech et al., 2008) and incubated for $20 \mathrm{hr}$ at $42^{\circ} \mathrm{C}$, followed by washing steps and finally scanning via an Agilent scanner. VACV gene expression was analyzed using a customized VACV array platform (VACGLa520445F) with 308 probes representing 219 genes that cover the genome of GLV-1h68, including the Renilla luciferase-Aequorea green fluorescent protein fusion gene, E.coli beta-glucuronidase, and 337 human or mouse "housekeeping" genes (393 probes) (Zhang et al., 2007). Fragmented, labeled aRNA $(6.5 \mu \mathrm{g})$ was hybridized to the VACV array platforms. After $16 \mathrm{hr}$ incubation in the hybridization oven at $45^{\circ} \mathrm{C}$, the arrays were washed and stained in the Fluidics station using the GeneChip ${ }^{\circledR}$ Hybridization, Wash, and Stain Kit. Finally, the arrays were scanned using the GeneChip ${ }^{\circledR}$ Scanner 3000 7G.

\section{Statistical analysis}

Human array transcriptional data were uploaded to the mAdb databank and analyzed using BRBArrayTools, developed by the Biometric Research Branch of the National Cancer Institute (Simon et al., 2007), or Partek Genomics Suite software as appropriate. VACV transcription was analyzed using Partek Genomics Suite software. Data retrieved from the VACV Affymetrix platform was normalized using a robust multichip average (RMA) approach. Unsupervised human data analysis was performed using the Stanford cluster program, and results were visualized with TreeView software (Eisen et al., 1998) or Partek Genomic Suite software as appropriate. Gene ratios were mean centered across all samples and displayed according to uncentered correlation algorithm and a complete linkage model. Ingenuity pathway analysis (IPA) was utilized to obtain biological insight in gene functions and interactions.

Time-dependent changes in human transcriptional patterns by different cell lines were identified by determination of time-dependent variances across noninfected samples. Time independent changes in human transcripts related to viral infection were identified by weighting variance in the infected sample as described in the results section. Correlation among different transcriptional parameters within the same or across platforms (human or VACV) were done according to Pearson's correlation analysis.

\section{Results}

Two previously characterized autologous cell lines (888MEL and 1936-MEL) derived from a patient with metastatic melanoma (Wang et al., 2006; Sabatino et al., 2008), were tested for permissiveness to the attenuated VACV GLV-1h68 strain and three wild-type VACV isolates: LIVP 1.1.1, LIVP 5.1.1, or LIVP 6.1.1 (MOI 0.01). 888-MEL was established when the cancer was still responsive to immunotherapy with interleukin-2 and tumor-infiltrating lymphocytes. More than a decade later 1936-MEL was generated from subsequent metastases when the cancer had become unresponsive to immunotherapy (Wang et al., 2006). Infected and noninfected control cells were tested 2, 6, 10, 24, and $48 \mathrm{hpi}$ for their transcriptional pattern.

\section{VACV-dependent changes in host cell transcription over time}

Using the human array platform, we first investigated in the two cell lines the culture-dependent kinetic changes in transcription, independent of infection (control samples). Unsupervised clustering segregated infected and respective uninfected samples together according to time in culture, suggesting that this parameter dominated the global transcriptional profile (Fig. 1A). To eliminate the dominant effect of time and highlight the transcriptional changes specific to VACV infection, we therefore applied a multistep filter (Fig. 1B). First, the standard deviation (SD) was calculated for each gene in uninfected samples $(2,10$, and $48 \mathrm{hpi}$ ); the analysis was performed separately for each cell line assuming cell-specific variation in gene expression during culture. A cut-off SD $>0.25$ was used that identified 8,300 genes stably expressed by uninfected; this set of genes was applied to recluster infected and uninfected 


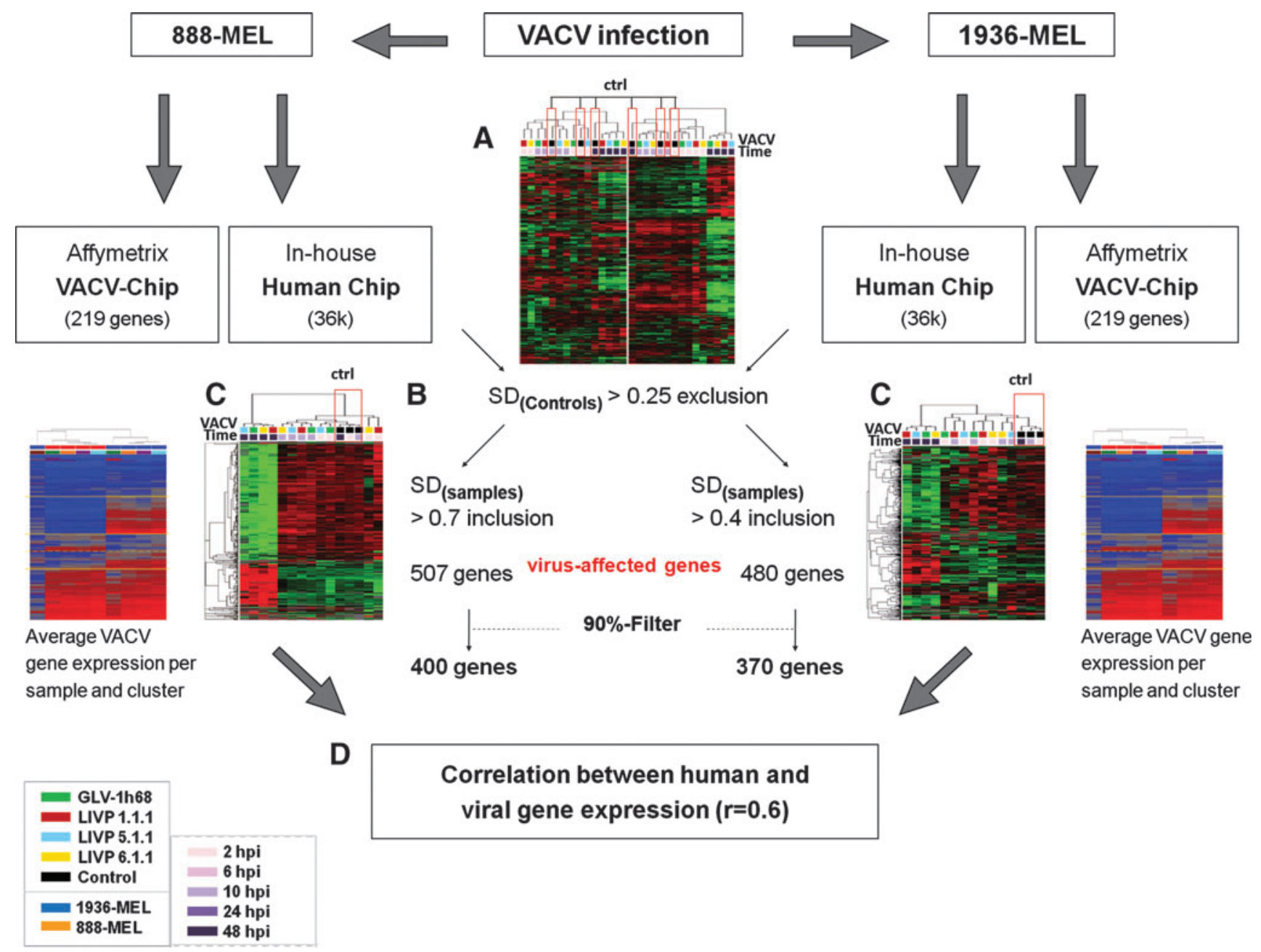

FIG. 1. Flow chart, describing the experiment for analysis of VACV-dependent changes in host cell transcription. 888-MEL and 1936-MEL cells were infected with four different VACV isolates at an MOI of 0.01 (GLV-1h68, LIVP 1.1.1, LIVP 5.1.1, and LIVP 6.1.1) in separate experiments or were treated with virus-free infection medium as negative control. Total RNA was amplified, purified, and labeled before generating transcriptional profiles via 36k human or custom-made VACV array platforms. (A) Expression profile of 888- and 1936-MEL cells after virus and mock infection at 2, 10, and 48 hr (filter 95\%, 2-fold). (B) Statistical approach to obtain a list of human genes affected by VACV infection specifically. (C) Human transcriptional profile after applying SD exclusion/inclusion method. (D) Correlation between VACV transcription and virusaffected host genes (Pearson correlation, $\mathrm{r}=0.6$ ). SD, standard deviation; ctr, control samples; hpi, hours post infection; VACV, Vaccinia virus; MOI, multiplicity of infection.

samples. The results still did not segregate uninfected from infected samples. Thus, we applied a second filter to include only transcripts with high SD in the infected samples, therefore, eliminating transcripts stably expressed by infected and uninfected cells. To identify a maximum number of infection-specific genes for each cell line, the SD cut-off was determined separately for each of them by a stepwise increase (intervals of 0.05) starting from a value of 0.3 and reclustering samples until uninfected samples built a separate cluster resulting in an SD $>0.7$ and $>0.4$ for 888 MEL and 1936-MEL respectively (Fig. 1C). For subsequent correlation analysis between human and viral gene expression, an additional filter was applied selecting genes whose expression values were present in at least $90 \%$ of samples (Fig. 1B and D). In the end, 400 and 370 infectionspecific genes were identified for 888-MEL and 1936-MEL respectively.
With the resulting gene pool, we investigated VACV effects on human gene transcription on the two autologous cell lines. The transcriptional profile at $48 \mathrm{hpi}$ revealed a dramatic alteration of expression of genes involved in broad cellular functions such as cell death, cellular growth and proliferation, protein synthesis and folding, and DNA replication, recombination, and repair (Supplementary Fig. S1; Supplementary Material available online at www.liebertonline .com/hgtb). These late effects were present independent of cell line or virus strain utilized (Fig. 1A and C). However, we previously observed that effects caused by VACV infection occur earlier when using wt isolates compared to GLV-1h68 (Supplementary Fig. S2); therefore, we focus the rest of the analysis on time-dependent, viral, and cell-specific changes.

Comparison of transcriptional patterns at 2 and $10 \mathrm{hpi}$ between the two cell lines revealed a clearer clustering according to time in 888-MEL and a less consistent pattern in 
1936-MEL, consistent with a less predictable responsiveness of this cell line to VACV infection in functional assays.

\section{VACV-dependent changes in viral gene transcription over time}

To characterize the transcriptional profile of the different VACV isolates, we compared viral gene expression at 2 and 10 hpi using a custom-made VACV array platform.

The 219 VACV genes represented in the VACV platform segregated in three array clusters and four main gene clus- ters $\mathrm{C} 1, \mathrm{C} 2, \mathrm{C} 3 \mathrm{a} / 3 \mathrm{~b}$, and C4 (Fig. 2A). VACV-infected arrays clustered according to time elapsed, following VACV infection while the uninfected arrays segregated separately. A clear separation between samples taken at 2 or 10 hpi was evident as also emphasized by principle component analysis (PCA) (Fig. 2B). GLV-1h68 contains three marker gene insertions (RUC-GFP: Renilla luciferase-green fluorescent protein (Aequorea) fusion; lacZ: $\beta$-galactosidase; and gus $A$ : $\beta$ glucoronidase cDNA), two of which are present in the VACV chip (RUC-GFP and gusA). At 10 hpi, both genes were expressed at high levels in cell line preparations infected with
A
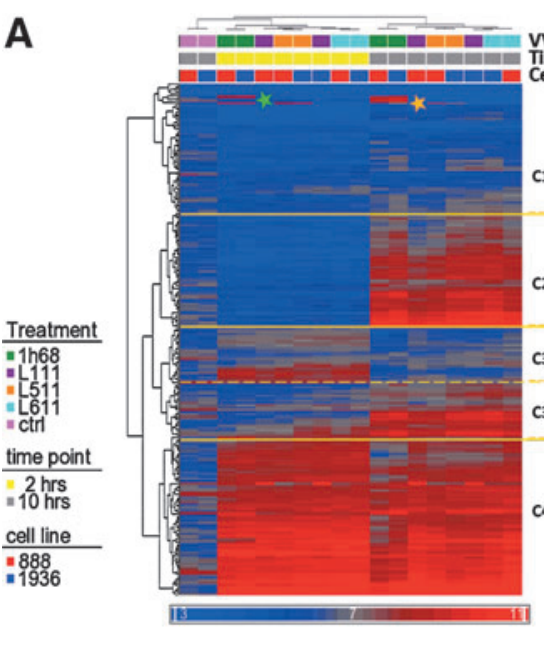

C
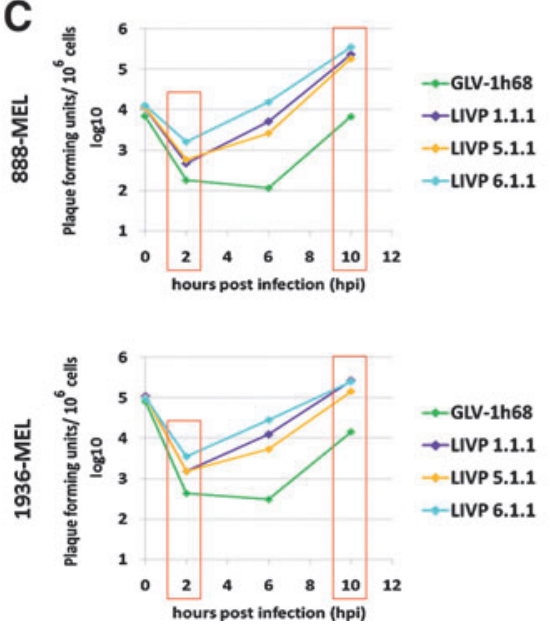

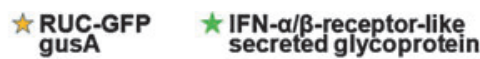
Time

- Similar (low) expression at 2 and $10 \mathrm{hpi}$; E/L genes - Genes interspersed throughout all categories (Wentry \& spread; c1 Structure \& assembly; DNA replication/RNAtranscription; Host interactions/immune modulations; other/unknown)

- Increased expression at $10 \mathrm{hpi}$ L genes C2 - Genes enriched in structure \& assembly class

\section{- Decreased expression at $10 \mathrm{hpi}$ E \& EL genes}

C3a - Genes enriched in DNA replication/RNA transcription class

- Increased expression at $10 \mathrm{hpi}$ L \& El genes

- Genes interspersed throughout all categories

- Similar (high) expression at 2 and $10 \mathrm{hpi}$ E \& El genes

c4 - Genes enriched in DNA replication/RNA transcription and Host interactions/immune modulations

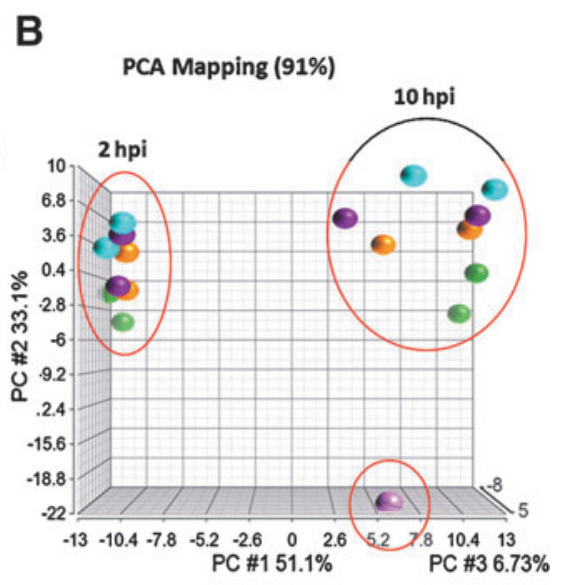

D
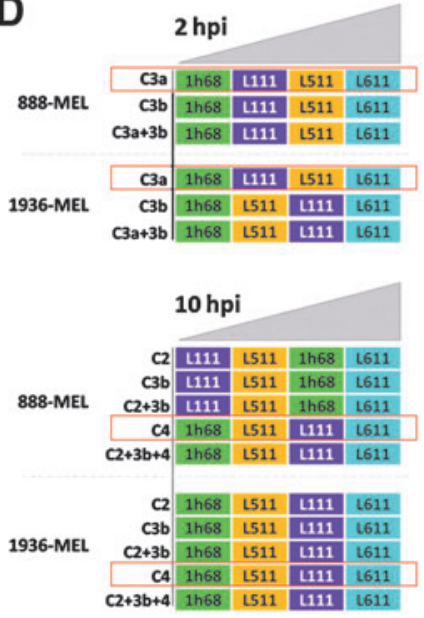

E
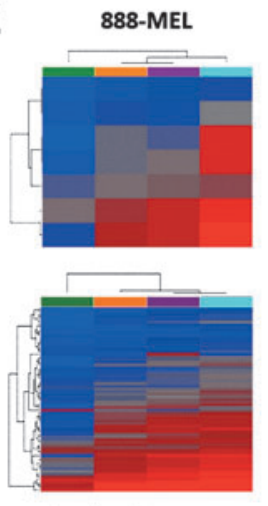

Treatment

-1h68

FIG. 2. VACV-isolate-specific changes in gene transcription over time and correlates with viral replication. 888-MEL and 1936-MEL cells were infected with four different VACV isolates (GLV-1h68, LIVP 1.1.1, LIVP 5.1.1, and LIVP 6.1.1) in separate experiments (MOI 0.01) or were treated with virus-free infection medium as negative control. Viral titers were determined by plaque assay and total RNA was isolated, amplified, purified, and labeled before generating transcriptional profiles via custom-made VACV array platforms. (A) Transcription profile including subcluster classification of four VACV isolates and negative control after 2 and $10 \mathrm{hr}$. (B) Principal components analysis (PCA, Partek Genomics Suite) of the VACV microarray data. Infection period and infectional status appeared to be a major source of variation, as indicated by red ellipsoids. The different colors specify the VACV isolate utilized. Green=GLV-1h68; purple=LIVP 1.1.1; yellow=LIVP 5.1.1, light blue=LIVP6.1.1; and pink = untreated controls. (C) VACV growth curves from 0 to 10 hpi. (D) Transcriptional ranking of VACV isolates. Transcription values were averaged considering the different time points and individual subclusters. Subsequently, viruses were ranked from lowest to highest mean gene transcription. (E) Hierarchical clustering of individual genes representing the transcriptional ranking, low to high: GLV-1h68, LIVP 5.1.1, LIVP 1.1.1, LIVP 6.1.1 or GLV-1h68, LIVP 1.1.1, LIVP 5.1.1, LIVP 6.1.1 in C3a (top) and C4 (bottom). C1, 2, 3a, 3b, 4, cluster 1, 2, 3a, 3b, 4; hpi, hours post infection; hrs, hours; VACV, Vaccinia virus; MOI, multiplicity of infection; RUC-GFP, Renilla luciferase-green fluorescent protein (Aequorea) fusion; gus $A$, $\beta$-glucoronidase. 
GLV-1h68 (Fig. 2A, yellow asterisk). Additionally, the viral interferon (IFN)- $\alpha / \beta$-receptor-like secreted glycoprotein (B19R, VACV Copenhagen), which is not present in full length in the genome of the wt clones, was expressed exclusively by GLV-1h68 (Fig. 2A, green asterisk).

The four gene clusters revealed a transcriptional pattern representative of the three promoter classes of VACV (early, early-late, and late). Moreover, we subdivided the open reading frame (ORF) products into five functional categories: VACV entry and spread, VACV structure and assembly, VACV DNA replication and RNA transcription, host interactions and immune modulations, and a final category representing genes with other or unknown functions. Genes present in cluster $\mathrm{C} 1$ showed similar low-expression levels at 2 and 10 hpi and included all functional categories. The only exceptions were the two marker genes RUC-GFP and gusA, $B 11 R$ with unknown function, and $B 19 R$, mentioned above. We found an enrichment of late genes in cluster C2 consistent with highly increased transcriptional levels at $10 \mathrm{hpi}$. Functional annotations revealed that these genes code predominantly structural components and enzymes involved in assembly of viral particles. Cluster C3a was enriched of early transcripts. Expectedly, proteins encoded by genes present in C3a regulate DNA replication and RNA transcription. An enrichment of early-late and late genes was found in cluster $\mathrm{C} 3 \mathrm{~b}$. The expressional pattern revealed notable expression at 2 hpi but this was more pronounced at the later time point. $\mathrm{C} 3 \mathrm{a}$ and $\mathrm{C} 3 \mathrm{~b}$ emerged as the clusters with the most heterogeneity among cell line preparations infected with different VACV constructs. Finally, cluster C4 represented early-late transcription with consistently high expression values especially at $2 \mathrm{hr}$. These genes mainly encoded proteins involved in modulation of the host immune response.

\section{$V A C V$ isolate-specific changes in transcription and correlation with viral replication}

VACV replication efficiency of GLV-1h68 and wt isolates. To characterize the different VACV wt isolates, we performed a time course analysis determining viral titers over time and comparing those with the well-characterized GLV-1h68 strain (Zhang et al., 2007). Using plaque assay analysis, we observed that all LIVP clones analyzed replicated more efficiently compared to GLV-1h68, especially at later time points. At $2 \mathrm{hr}$ the viruses ranked according to their replication from lowest to highest titer as follows: GLV1h68, LIVP 5.1.1/LIVP 1.1.1, and LIVP 6.1.1 (Fig. 2C). The ranking was consistent for both melanoma cell lines. With progression of the infection, the number of plaque-forming units became closer among all LIVP isolates with a widening gap compared to GLV-1h68.

Ranking of VACV gene transcription level and correlation with VACV replication. To compare viral transcription with replication according to plaque-forming units, we first ranked viral isolates according to their overall transcriptional activity. Within each gene cluster category (Fig. 2A), we averaged the intensity values of all genes for each virus at each time point. Averaged VACV gene expression was ranked from lowest to highest for each virus. The subclusters $\mathrm{C} 3 \mathrm{a}$ and $\mathrm{C} 3 \mathrm{~b}$ were considered separately and in combination as representative of early gene transcription
(2 hpi). The subclusters C2, C3b, C4, C2/C3b, and C2/C3b/ $\mathrm{C} 4$ were analyzed to assess late gene expression (10 hpi).

VACV isolate-specific changes in transcription at $2 \mathrm{hpi}$. We focused subsequent analyses on gene subclusters, revealing the most pronounced differences among LIVP 1.1.1, LIVP 5.1.1, LIVP 6.1.1, and GLV-1h68 at 2 hpi. At this time point, GLV-1h68 displayed the lowest mean levels of gene transcription in all subclusters analyzed, whereas LIVP 6.1.1 showed the highest values at $2 \mathrm{hpi}$ independent of cell line tested (Fig. 2D, top). LIVP 5.1.1 and LIVP 1.1.1 displayed comparable intermediate transcription levels and were, therefore, ranked together (rank $1=$ LIVP 6.1.1; rank $2=$ LIVP 5.1.1 and LIVP 1.1.1; and rank 3=GLV-1h68). As mentioned above, cluster $\mathrm{C} 3 \mathrm{a}$ was enriched of early transcripts. Although cluster $\mathrm{C} 4$ also reflects high early expression levels, there were no clear differences among VACV isolates at $2 \mathrm{hpi}$. Based on the equal gene expression of all VACV isolates in cluster C4 at $2 \mathrm{hpi}$, this cluster appeared to be ineligible to point out differences between the viruses. For this reason, gene cluster $\mathrm{C} 3 \mathrm{a}$ emerged as the most representative subcluster to study VACV at $2 \mathrm{hpi}$.

Subsequently, seven genes from cluster C3a, whose expression corresponded with the overall early transcriptional ranking (rank 1-3, see above) were identified: F15L, G2R, G5R, D9R, A5R, A20R, and A24R. These genes encode subunits of the DNA-dependent RNA polymerase, DNA polymerase processivity factor, NTP phosphorylase, late transcription elongation factor, and a viral membrane formation protein. Hierarchical clustering based on these seven genes was used to test whether they could segregate experiments according to VACV-specific gene transcription (Fig. $2 \mathrm{E}$, top). The early viral gene transcription ranking (rank 1-3) was consistent with replication according to plaque-forming units at 2 hpi.

VACV isolate-specific changes in transcription at $10 \mathrm{hpi}$. Accordingly, we concentrated on subclusters pointing at differences among viral isolates at $10 \mathrm{hpi}$. At this time point, gene transcription intensities in clusters $\mathrm{C} 1, \mathrm{C} 2$, and $\mathrm{C} 3 \mathrm{~b}$ were higher for GLV-1h68 when compared to LIVP 5.1.1. In other words, viral gene transcription ranking (rank 1-3) was inconsistent with replication according to plaque-forming units at 10 hpi in these clusters (Fig. 2D, bottom). However, gene cluster $\mathrm{C} 4$ displayed strong distinctions in transcription intensity, comparing GLV-1h68 with the different wt clones, in accordance with replication data at $10 \mathrm{hpi}$. In both melanoma cell lines the lowest mean transcription was observed for GLV-1h68, followed by LIVP 5.1.1 and LIVP 1.1.1 and finally by LIVP 6.1 .1 (Fig. 2D, bottom). Consequently, we considered cluster $\mathrm{C} 4$ as representative of viral replication according to plaque-forming units and used it for further comparisons at $10 \mathrm{hpi}$.

We identified $32 \mathrm{VACV}$ genes in C4 whose expression followed the previously discussed ranking in both cell lines. Self organizing reclustering of $10 \mathrm{hpi}$ microarray samples based on these genes demonstrated a clear gradient in transcription by the VACV isolates (Fig. 2E, bottom). Furthermore, in accordance with VACV replication data, GLV-1h68-transcription demonstrated a clear separation from all wt isolates. Functional annotations revealed that almost half of the 32 genes were involved in DNA replication and RNA 
transcription and about one third in host interactions. Among the former, we found genes whose products act in deoxyribonucleotid triphosphate (dNTP) synthesis $(F 2 L, J 2 L, I 4 L)$, replication initiation, and continuance (primase/NTPase activity, ssDNA-binding), as well as components of the DNAdependent RNA polymerase.

\section{Correlates between VACV and host cell transcription}

To explore the relationship between host cell transcription and viral replication, we correlated viral and human gene expression at different time points.

At high multiplicity of infection (MOI >5), VACV gene transcription levels are homogeneous according to the different temporal classes, independent of the VACV isolate utilized. We, therefore, adopted low MOI to increase the chance that subtle differences in the kinetics of transcriptional activation among viral isolates could segregate infectivity among different viruses.

We first characterized the correlation between viral early and human early gene transcription ( $2 \mathrm{hpi}$ ) and identified seven VACV genes in cluster C3a (viral arrays) that were most representative of early events; we will refer to these seven genes as viral replication indicators (VRI). The expression values of the seven VRIs were averaged for each sample. The samples in turn were ranked according to average transcription level of the VRI (Fig. 2D). Comparison between the VRI and human gene expression were performed by Pearson's correlation. We identified 114 human genes strongly correlating $(r \geq 0.6)$ with VRI. IPA revealed that these genes belong to the following networks: post-translational modification, free radical scavenging, gene expression, cell death, and cellular growth and proliferation. The top molecular functions were cell cycle, cellular movement, development, growth and proliferation, and cell-to-cell signaling.

We then investigated if human early gene transcription could predict VACV intermediate/late transcription. As discussed above, $32 \mathrm{VACV}$ genes from cluster $\mathrm{C} 4$ seemed to represent VACV isolate-specific intermediate/late replication-associated events. Sample-specific transcription value averages of these 32 genes were sorted according to the ranking of their transcription level (Fig. 2D). After arranging the respective human expression table accordingly, Pearson's correlation analyses were applied to determine individual correlation coefficients. Using a cut-off of $r=0.6$, we identified 84 human early genes that correlate with VACV intermediate/late gene transcription. We found that the majority of these 84 genes were involved in cell death, cell cycle, lipid metabolism, small molecule biochemistry, and cellular development. Comparing top canonical pathways of human correlates with VACV early and intermediate/late transcription, overlapping pathways were observable, such as D glutamine and D glutamate signaling, anti-proliferative role of TOB in T cell signaling, and cell cycle checkpoint regulation (Fig. 3A and B). Even though the IPA results have to be considered with caution, due to the low number of genes, we found an intercept of 14 focus molecules for early and late correlates present in the network: Post-translational modification, free radical scavenging, and gene expression (Fig. 3C and D). Further, there was an overlap of 18 focus molecules in the network involving cell death, lipid metabolism, and small molecule biochemistry.

\section{Prediction of VACV replication}

Based on the early transcriptional correlates identified in the two melanoma cell lines, we tested their predictive strength in an independent data set, including different cell lines and different viral constructs. Chen et al. (2011) described that virus replication efficiency is linked to the number of transgene insertions in a promoter-strengthdependent manner using a series of recombinant VACV strains, including GLV-1h68. Further, they demonstrated a correlation between replication efficiency of these VACV strains with antitumor efficacy and virulence. Therefore, this series of recombinant viruses appeared well-suited to test our hypothesis that a set of human genes may influence viral replication efficiency and/or permissiveness to VACV infection. A human breast cancer (GI101A) and a colon carcinoma (HT29) cell line were individually infected with GLV-1h68 and five VACV recombinants (GLV-1h70, GLV1h71, GLV-1h72, GLV-1h73, and GLV-1h74). Samples were taken at 2 hpi. Infections, RNA isolation, and microarray analyses were performed identically to the previous set.

For the prediction, we assumed that the transcriptional pattern of the seven VRIs (Fig. 2E; F15L, G2R, G5R, D9R, $A 5 R, A 20 R$, and $A 24 R)$ reflects viral replication. Further, human melanoma genes that correlated with the average of the VRIs ( $r \geq 0.5$ ) were assumed indicative of viral replication. We applied the same analytic criteria to the data set generated for GI101A and HT29. Gene transcription values of the VRIs in GI101A and HT29 were averaged and arranged according to value. Then, averaged viral gene expression was correlated with the respective human gene transcription (Pearson's Correlation). Correlates were included by the following criteria: (1) $\mathrm{r}$ in melanoma cell lines $\geq 0.5$ (absolute value); (2) $\mathrm{r}$ in all cell lines must have the same algebraic sign (positive or negative); and (3) $r$ in GI101A and HT29 has to be either at least moderate ( $r$ between -0.5 and -0.3 , or between 0.3 and 0.5 ) in both cases, or, if one correlation is weak in one cell line it must be strong in the other one.

We found 10 genes that matched these criteria (Fig. 4A and B) as potential human indicators for viral replication efficiency. The strongest positive correlations were observed for lantibiotic synthetase component C-like 2 (LANCL2) and heterogeneous nuclear ribonucleoprotein $\mathrm{L}$ (HNRNPL). Sortin nexin 17 (SNX17) transcription correlated strongly with viral early transcription in HT29 and the melanoma cell lines and revealed a moderate correlation within GI101A. The strongest negative correlations were found for ATP synthase, $\mathrm{H}+$ transporting, mitochondrial Fo complex, subunit B1 (ATP5F1), and chemokine (C-C motif) ligand 5 (CCL5).

\section{Discussion}

Since the approval of the first oncolytic virus for cancer therapy in 2005 (Garber, 2006), huge progress has been made in engineering oncolytic viruses with enhanced safety, specificity, oncolytic efficiency, and reduced toxicity (Wong et al., 2010; Hernandez-Alcoceba, 2011). Despite the remarkable progress in oncolytic VACV-therapy, pre-existing immunity in smallpox vaccinated subjects is still a concern since neutralizing antibodies could limit the efficacy of the therapy. A preformed protection against poxviruses is 
A

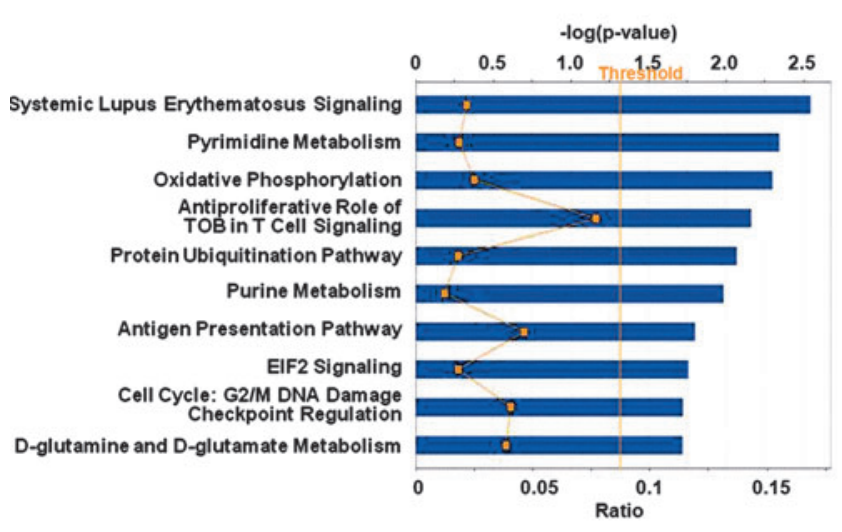

C

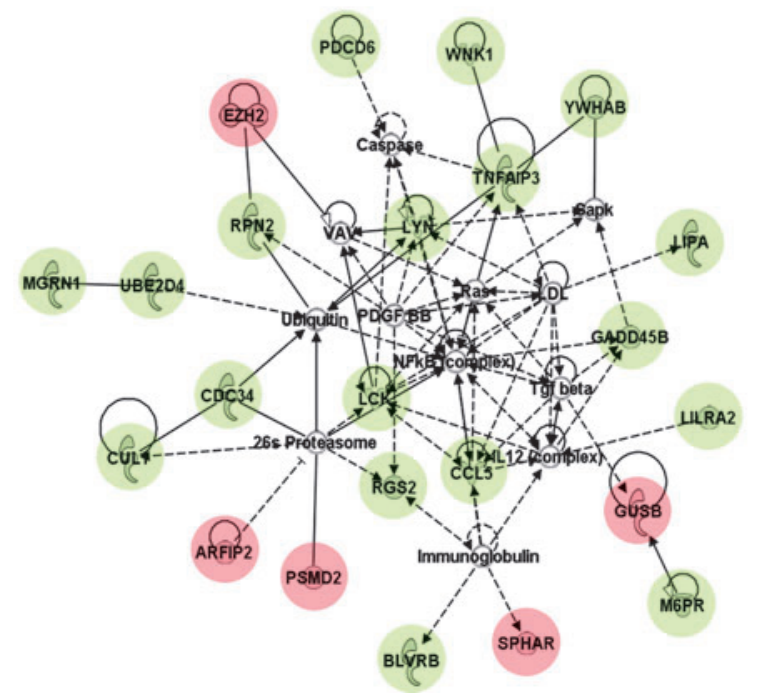

B

10 hpi (VACV) vs. 2 hpi (h)

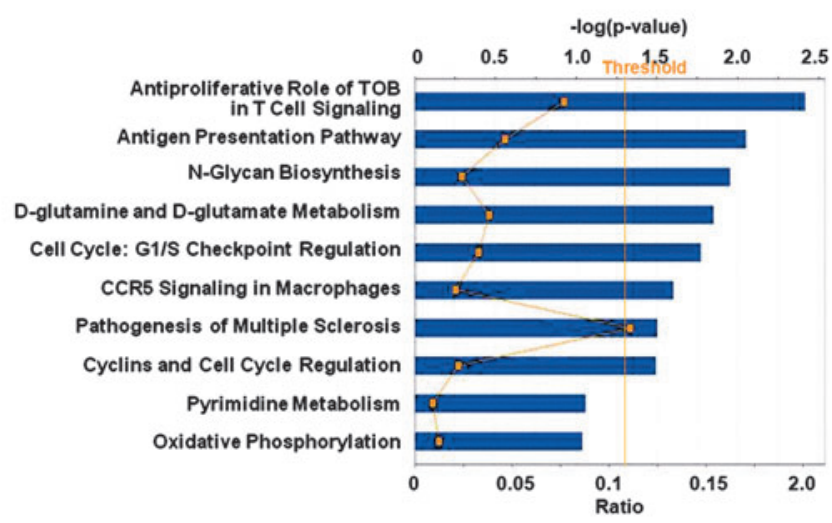

D

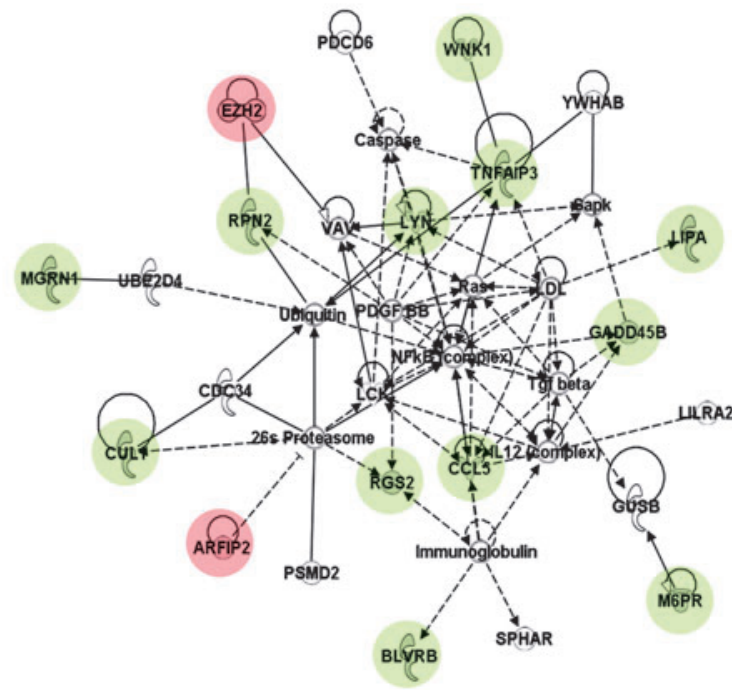

FIG. 3. Pathway analyses of correlates between VACV and host cell transcription. Canonical pathways and top networks were identified by ingenuity pathway analysis (IPA). (A) Top canonical pathways of 114 early human correlates with averaged VACV early gene transcription. (B) Top canonical pathways of 84 early human correlates with averaged VACV intermediate/late gene transcription. (C, D) Network: post-translational modification, free radical scavenging, and gene expression revealed an overlap of 14 genes out of an intercept of 66 genes between early (C) and intermediate/late (D) correlates. Red circles, positive correlation; green circles, negative correlation; hpi, hours post infection; h, human; VACV, Vaccinia virus.

particularly problematic for systemic treatment. Current research targeting this issue has focused on alternative routes of vaccine delivery (i.e., intratumoral), temporary immunosuppression, and immune evasion via carrier cell delivery (Guo et al., 2010) as well as dose escalation.

In addition, the factors contributing to virus-mediated tumor regression and therapeutic outcome are not yet fully understood. Immune-mediated oncolysis following viral infection (Prestwich et al., 2009), destruction of the tumor vasculature (Frentzen et al., 2009; Tysome et al., 2009), and direct viral oncolysis (Weibel et al., 2011) are debated as possible mechanisms. We previously observed that the kinetics of in vitro viral replication correlates with in vivo oncolytic function (Worschech et al., 2009); therefore, in this study we investigated whether parameters could be investigated that could predict VACV replication in vitro.

The LIVP-derived oncolytic VACV strain GLV-1h68 (Zhang et al., 2007) has been characterized extensively, and its antitumor effect has been demonstrated for many solid cancers (Lin et al., 2007; Zhang et al., 2007; Kelly et al., 2008; Yu et al., 2009a; Yu et al., 2009b; Gentschev et al., 2010; Gentschev et al., 2011). Recently, a series of VACV wt clones, derived from the mixed population of the GLV-1h68 parental strain, were characterized regarding replication efficiency, toxicity, and therapeutic effect in nude mice (unpublished). Three isolates that showed natural attenuation due to point mutations and deletions were used in this study (LIVP 1.1.1, LIVP 5.1.1, and LIVP 6.1.1.).

We, therefore, analyzed in vitro transcription of distinct viral preparations to identify potential markers of permissiveness of cancer cells to VACV infection. Although cell culture provides enormous benefits, its limitations have to be considered. It has been documented previously that time in culture can alter the gene expression profile of cells drastically (Zaitseva et al., 2006; Neumann et al., 2010). Accordingly, we observed that the gene expression profile of 
A

\begin{tabular}{c|cccc} 
NAME & 888-MEL & 1936-MEL & Gl101A & HT29 \\
\hline \hline LANCL2 & 0.67 & 0.85 & 0.53 & 0.62 \\
HNRNPL & 0.59 & 0.93 & 0.56 & 0.73 \\
SNX17 & 0.63 & 0.84 & 0.43 & 0.80 \\
TMED1 & 0.87 & 0.77 & 0.21 & 0.58 \\
TXNRD3 & 0.90 & 0.88 & 0.28 & 0.47 \\
ZNF69 & 0.66 & 0.79 & 0.36 & 0.34 \\
\hline CCL5 & -0.83 & -0.94 & -0.42 & -0.44 \\
ATP5F1 & -0.69 & -0.73 & -0.30 & -0.67 \\
ID2 & -0.85 & -0.84 & -0.55 & -0.23 \\
DEK & -0.90 & -0.62 & -0.48 & -0.24
\end{tabular}

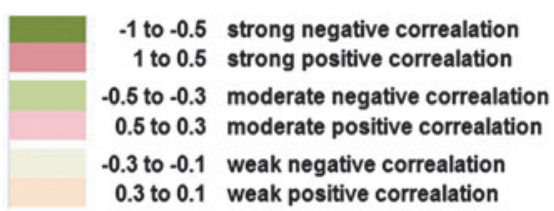

HT29,

GI101A

V isolate

$=\mathrm{GLV}-1 \mathrm{~h} 68$

- GLV-1h70

- GLV-1h71

$=$ GLV-1h72

$=$ GLV-1h73

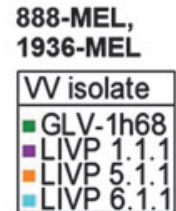

B

888-MEL

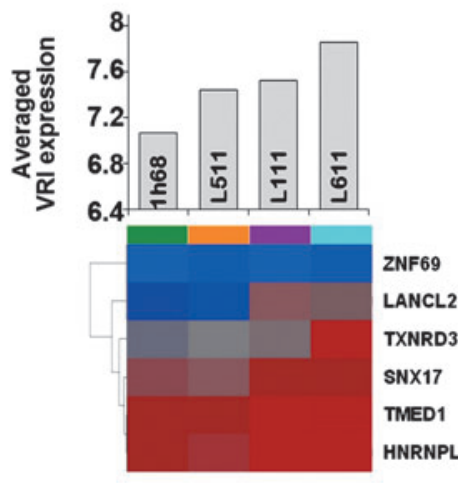

Gl101A

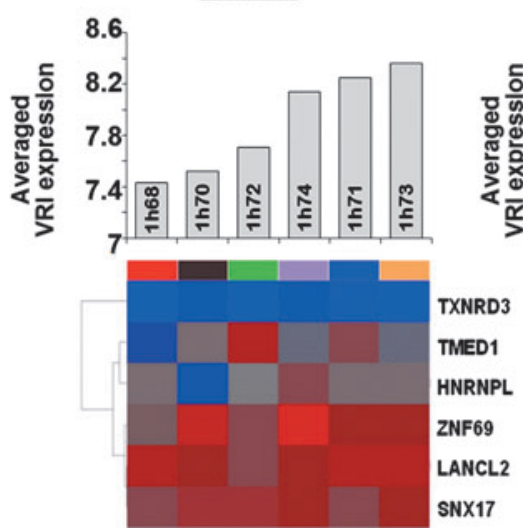

1936-MEL

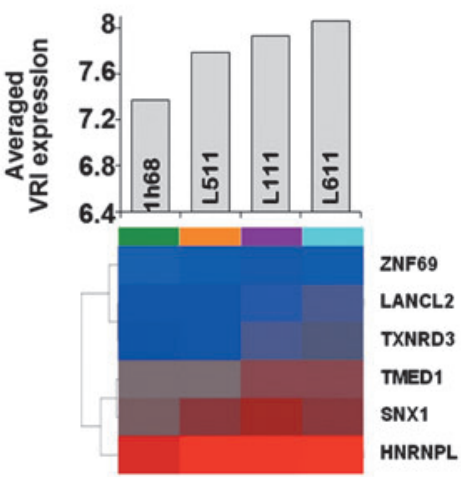

$\underline{\mathrm{HT} 29}$

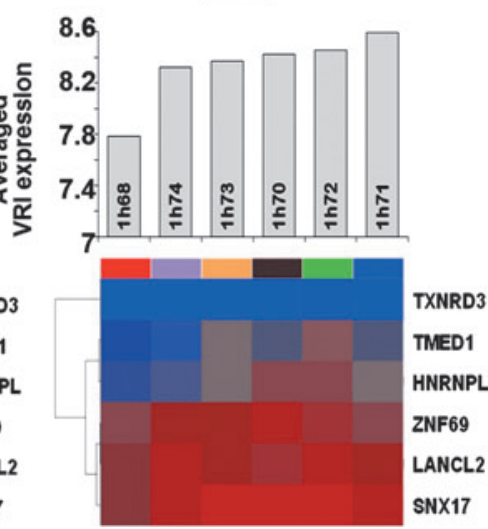

FIG. 4. Prediction of viral replication in an independent dataset. GI101A and HT29 cells were infected with six different VACV isolates (GLV-1h68, GLV-1h70, GLV-1h71, GLV-1h72, GLV-1h73, and GLV-1h74) in separate experiments (MOI 0.01). Total RNA was isolated, amplified, purified, and labeled before generating transcriptional profiles via custom-made VACV array platforms or human whole genome (36k) array platforms. Predictor genes were determined by the following criteria: $\mathrm{r}$ in melanoma cell lines $\geq 0.5$ (absolute value); $r$ in all cell lines has to have same direction (positive or negative); and $\mathrm{r}$ in GI101A and HT29 has to be either at least moderate in both cases or, if one correlation is weak in one cell line, it must be strong in the other one. (A) Ten predictors of viral replication were determined by Pearson Correlation. (B) Heat map of the six predictive positive human correlates at $2 \mathrm{hpi}$ in 888-MEL, 1936-MEL, GI101A, and HT29; averaged viral gene expression of seven VRIs at $2 \mathrm{hpi}$ from cluster C3a (bar graphs). VRI, viral replication indicators.

uninfected control samples greatly varied according to culture time. This variation resulted in a lack of segregation between infected and uninfected samples. However, a multistep filter could determine a list of genes affected specifically by VACV infection.

It has been reported that VACV infection causes major alterations in cell function and metabolism (Buller and Palumbo, 1991), and results in a massive interference with host DNA/RNA and protein synthesis (Moss, 2007). Guerra et al. (2003) classified mRNAs that showed an altered expression pattern after VACV infection of HeLa cells into distinct groups depending on their kinetics of gene activation or repression. Concordantly, we identified distinct categories of virus-affected genes involved in broad cellular functions such as cell death; cellular growth and proliferation; protein synthesis and folding; and DNA replication, recombination, and repair. Expectedly, these genes revealed a drastic up or down regulation after $48 \mathrm{hr}$ that was similar, independent of viral strain utilized. We, therefore, postulate that initial events, at an early stage of infection, can provide more valuable information regarding permissiveness of a given cell line to VACV infection. For this reason, we focused our analysis on data obtained at 2 and $10 \mathrm{hpi}$.

In parallel to host gene expression, viral gene expression was analyzed to get better insight over the interplay between VACV and host. Furthermore, VACV gene expression was evaluated as a parameter representative of viral replication. Recently, we showed that GFP-marker gene expression of GLV-1h68 correlates with the respective viral copy number in A375, DU-145, and A549 cells (Ascierto et al., 2011). Using a customized VACV array platform, we identified a group of genes that could be classified as early-stage genes by their gene transcriptional pattern. Comparing the average gene expression of those early genes within the different VACV isolates, we found that LIVP 6.1.1 showed the highest mean levels of gene transcription, followed by LIVP 1.1.1 and LIVP 5.1.1, and finally by GLV-1h68. Plaque assay analysis revealed comparable results when looking at viral titers. We therefore consider early gene expression as an appropriate variable to represent viral replication.

Little is known about how interactions between VACV and host contribute to the permissiveness of a host cell to 
infection. Poxviruses like VACV carry out their entire life cycle, including DNA replication, transcription, and translation in the cytoplasm of its host cell. In particular, it has been shown that certain cellular factors are required for postreplicative transcription (Zhu et al., 1998; Wright et al., 2001; Katsafanas and Moss, 2004; Knutson et al., 2006). However, a participation of host factors in viral early gene transcription or DNA replication remains poorly understood. Even though VACV provides all factors and enzymes directly required for DNA and early RNA synthesis packaged within the virus particle, host gene products might affect efficiency and outcome of the syntheses by either providing a framework or through facilitating molecular interactions.

In this study, we identified several parameters that may be useful for the ultimate understanding of how VACV and host cells interact: 1) we observed that a subset of VACV early-late transcripts is mostly associated with variability in permissiveness of cell lines to VACV infection; 2) among them we identified 7 and 32 transcripts (viral replication indicators, or VRIs) that correlated strictly with viral replication at 2 and $10 \mathrm{hpi}$, respectively, as judged by the functional plaqueforming units assay; 3) further we identified a subset of 114 and 84 human transcripts whose expression tightly correlated with that of the respective VRIs at 2 and $10 \mathrm{hpi}$; 4) finally, 10 of the 114 genes could be validated as predictors of VACV replicative efficiency in an independent data set.

Out of the 7 VRIs (F15L, G2R, G5R, D9R, A5R, A20R, and $A 24 R)$ used for correlation analysis, five are clearly involved in viral replication or transcription. Among them we identified subunits of the DNA-dependent RNA polymerase, DNA polymerase processivity factor, NTP phosphorylase, and a late transcription elongation factor. It has been reported previously that the DNA polymerase processivity factor A20 interacts with other VACV DNA replication and transcription-relevant proteins (D4, D5, E9, and H5) (Van Vliet et al., 2009).

The 114 human genes correlating with VRI expression represented cellular involvement in post-translational modification, free radical scavenging, gene expression, cell death, cell-to-cell signaling, cell cycle, and cellular growth and proliferation.

Finally, the 10 genes that could be validated in an independent data set included heterogeneous nuclear ribonucleoprotein L (HNRNPL). Proteins of the hnRNP family feature wide-ranging functions (Chaudhury et al., 2010; Dreyfuss et al., 1993; Krecic and Swanson, 1999) and shuttle between the nucleus and the cytoplasm (Pinol-Roma and Dreyfuss, 1992). It has been reported previously that members of the hnRNP family may participate in vaccinia virus late transcription (Wright et al., 2001; Dellis et al., 2004) as well as in the replication of other viruses (Li and Nagy, 2011). Functions assigned to hnRNP L include, but are not limited to, an involvement in transport of intronless mRNAs (Liu and Mertz, 1995; Guang et al., 2005), a role in hepatitis C virus (HCV) internal ribosome entry site (IRES)-mediated mRNA translation (Hwang et al., 2009), as well as in mRNA stability. Therefore, it is conceivable that hnRNP proteins might influence vaccinia virus replication efficacy and thus permissiveness of a host cell to VACV infection. Furthermore, strong negative correlations were detected for ATP synthase, $\mathrm{H}^{+}$transporting, mitochondrial $\mathrm{F}_{\mathrm{o}}$ complex, subunit B1 (ATP5F1), and chemokine (C-C motif) ligand 5
(CCL5). The down-regulation of mitochondrial ATPase expression is considered as a hallmark of several human carcinomas (Willers and Cuezva, 2011; Cuezva et al., 2002). Therefore, one could speculate an association between degree of ATP5F1 down-regulation and permissiveness to VACV treatment. The chemotactic cytokine CCL5/RANTES, (regulated upon activation, normal T cell expressed and secreted) in turn, is known for its antiviral activity (Glass et al., 2003). Yu et al. (2009a) demonstrated through immunerelated protein antigen profiling a down-regulation of RANTES in pancreatic xenografts in nude mice upon GLV-1h68 infection. An inverse correlation between VACV replication and CCL5 expression could therefore indicate an immune evasion strategy, thereby providing a conductive environment for viral amplification.

In summary, we identified a set of human genes that are likely to affect viral replication. Although more comprehensive studies, including an increased sample size, fine tuning of the applied statistics, and application of different confirmation methods appear to be appropriate and will be needed to confirm these findings. The discovery of host factors that influence viral replication could provide valuable information about host cell permissiveness to oncolytic virotherapy and therapeutic efficiency. Moreover, it could help identify possible biomarkers that predict response to VACV treatment, eventually.

\section{Acknowledgments}

The authors appreciate the excellent technical assistance of Mr. T. Trevino (Genelux Corporation), Mrs. H. Liu, Mrs. R. Shi, and Mr. J. Chen (all three from National Institutes of Health). We thank Genelux Corporation for provision of the customized vaccinia virus chips (by Affymetrix). Furthermore, this work was supported by a research grant from Genelux Corporation (R\&D facility in San Diego, CA) awarded to the University of Wuerzburg, Germany.

\section{Disclosure Statement}

N.G. Chen, Q. Zhang, Y.A. Yu, R.J. Aguilar, and A.A. Szalay are employees of Genelux Corporation and have personal financial interests in Genelux Corporation. J. Reinboth, special volunteer at NIH, is a graduate student in Dr. Szalay's laboratory in the Department of Biochemistry, University of Würzburg, Germany, and is supported by a graduate stipend and foreign travel grant from Genelux Corporation.

\section{References}

Ascierto, M.L., Worschech, A., Yu, Z., et al. (2011). Permissivity of the NCI-60 cancer cell lines to oncolytic Vaccinia Virus GLV-1h68. BMC Cancer 11, 451.

Broyles, S.S., and Knutson, B.A. (2010). Poxvirus transcription. Future Virol. 5, 639-650.

Buller, R.M., and Palumbo, G.J. (1991). Poxvirus pathogenesis. Microbiol. Rev. 55, 80-122.

Buller, R.M., Smith, G.L., Cremer, K., et al. (1985). Decreased virulence of recombinant vaccinia virus expression vectors is associated with a thymidine kinase-negative phenotype. Nature 317, 813-815.

Chaudhury, A., Chander, P., and Howe, P.H. (2010). Heterogeneous nuclear ribonucleoproteins (hnRNPs) in cellular 
processes: Focus on hnRNP E1's multifunctional regulatory roles. RNA 16, 1449-1462.

Chen, N.G., Yu, Y.A., Zhang, Q., and Szalay, A.A. (2011). Replication efficiency of oncolytic vaccinia virus in cell cultures prognosticates the virulence and antitumor efficacy in mice. J. Transl. Med. 9, 164.

Cuezva, J.M., Krajewska, M., De Heredia, M.L., et al. (2002). The bioenergetic signature of cancer: a marker of tumor progression. Cancer Res. 62, 6674-6681.

Dellis, S., Strickland, K.C., Mccrary, W.J., et al. (2004). Protein interactions among the vaccinia virus late transcription factors. Virology 329, 328-336.

Dreyfuss, G., Matunis, M.J., Pinol-Roma, S., and Burd, C.G. (1993). hnRNP proteins and the biogenesis of mRNA. Annu. Rev. Biochem. 62, 289-321.

Eisen, M.B., Spellman, P.T., Brown, P.O., and Botstein, D. (1998). Cluster analysis and display of genome-wide expression patterns. Proc. Natl. Acad. Sci. U.S.A. 95, 14863-14868.

Falkner, F.G., and Moss, B. (1990). Transient dominant selection of recombinant vaccinia viruses. J. Virol. 64, 3108-3111.

Flexner, C., Hugin, A., and Moss, B. (1987). Prevention of vaccinia virus infection in immunodeficient mice by vectordirected IL-2 expression. Nature 330, 259-262.

Frentzen, A., Yu, Y.A., Chen, N., et al. (2009). Anti-VEGF singlechain antibody GLAF-1 encoded by oncolytic vaccinia virus significantly enhances antitumor therapy. Proc. Natl. Acad. Sci. U.S.A. 106, 12915-12920.

Garber, K. (2006). China approves world's first oncolytic virus therapy for cancer treatment. J. Natl. Cancer Inst. 98, 298-300.

Gentschev, I., Donat, U., Hofmann, E., et al. (2010). Regression of human prostate tumors and metastases in nude mice following treatment with the recombinant oncolytic vaccinia virus GLV-1h68. J. Biomed. Biotechnol. 2010, 489759.

Gentschev, I., Muller, M., Adelfinger, M., et al. (2011). Efficient colonization and therapy of human hepatocellular carcinoma (HCC) using the oncolytic vaccinia virus strain GLV-1h68. PLoS One 6, e22069.

Glass, W.G., Rosenberg, H.F., and Murphy, P.M. (2003). Chemokine regulation of inflammation during acute viral infection. Curr. Opin. Allergy Clin. Immunol. 3, 467-473.

Guang, S., Felthauser, A.M., and Mertz, J.E. (2005). Binding of hnRNP L to the pre-mRNA processing enhancer of the herpes simplex virus thymidine kinase gene enhances both polyadenylation and nucleocytoplasmic export of intronless mRNAs. Mol. Cell. Biol. 25, 6303-6313.

Guerra, S., Lopez-Fernandez, L.A., Pascual-Montano, A., et al. (2003). Cellular gene expression survey of vaccinia virus infection of human HeLa cells. J. Virol. 77, 6493-6506.

Guo, Z.S., Parimi, V., O'Malley, M.E., et al. (2010). The combination of immunosuppression and carrier cells significantly enhances the efficacy of oncolytic poxvirus in the pre-immunized host. Gene Ther. 17, 1465-1475.

Guo, Z.S., Thorne, S.H., And Bartlett, D.L. (2008). Oncolytic virotherapy: molecular targets in tumor-selective replication and carrier cell-mediated delivery of oncolytic viruses. Biochim. Biophys. Acta. 1785, 217-231.

Hernandez-Alcoceba, R. (2011). Recent advances in oncolytic virus design. Clin. Transl. Oncol. 13, 229-239.

Hwang, B., Lim, J.H., Hahm, B., et al. (2009). hnRNP L is required for the translation mediated by HCV IRES. Biochem. Biophys. Res. Commun. 378, 584-588.

Izmailyan, R., and Chang, W. (2008). Vaccinia virus WR53.5/ F14.5 protein is a new component of intracellular mature virus and is important for calcium-independent cell adhesion and vaccinia virus virulence in mice. J. Virol. 82, 10079-10087.

Jin, P., Zhao, Y., Ngalame, Y., et al. (2004). Selection and validation of endogenous reference genes using a high throughput approach. BMC Genomics 5, 55.

Katsafanas, G.C., and Moss, B. (2004). Vaccinia virus intermediate stage transcription is complemented by Ras-GTPaseactivating protein $\mathrm{SH} 3$ domain-binding protein (G3BP) and cytoplasmic activation/proliferation-associated protein ( $\mathrm{p} 137$ ) individually or as a heterodimer. J. Biol. Chem. 279, 5221052217.

Kelly, K.J., Woo, Y., Brader, P., et al. (2008). Novel oncolytic agent GLV-1h68 is effective against malignant pleural mesothelioma. Hum. Gene Ther. 19, 774-782.

Knutson, B.A., Liu, X., Oh, J., and Broyles, S.S. (2006). Vaccinia virus intermediate and late promoter elements are targeted by the TATA-binding protein. J. Virol. 80, 6784-6793.

Krecic, A.M., and Swanson, M.S. (1999). hnRNP complexes: composition, structure, and function. Curr. Opin. Cell Biol. 11, 363-371.

Lee, M.S., Roos, J.M., Mcguigan, L.C., et al. (1992). Molecular attenuation of vaccinia virus: mutant generation and animal characterization. J. Virol. 66, 2617-2630.

Li, Z., and Nagy, P.D. (2011). Diverse roles of host RNA binding proteins in RNA virus replication. RNA Biol. 8, 305-315.

Lin, S.F., Yu, Z., Riedl, C., et al. (2007). Treatment of anaplastic thyroid carcinoma in vitro with a mutant vaccinia virus. Surgery 142, 976-983; discussion 976-983.

Liu, X., and Mertz, J.E. (1995). HnRNP L binds a cis-acting RNA sequence element that enables intron-dependent gene expression. Genes Dev. 9, 1766-1780.

McCart, J.A., Ward, J.M., Lee, J., et al. (2001). Systemic cancer therapy with a tumor-selective vaccinia virus mutant lacking thymidine kinase and vaccinia growth factor genes. Cancer Res. 61, 8751-8757.

Moss, B. (2007). Poxviridae: The Viruses and their Replication. (Lippincott Williams \& Wilkins, Philadelphia).

Neumann, E., Riepl, B., Knedla, A., et al. (2010). Cell culture and passaging alters gene expression pattern and proliferation rate in rheumatoid arthritis synovial fibroblasts. Arthritis Res. Ther. 12, R83.

Pinol-Roma, S., and Dreyfuss, G. (1992). Shuttling of pre-mRNA binding proteins between nucleus and cytoplasm. Nature 355, 730-732.

Prestwich, R.J., Errington, F., Diaz, R.M., et al. (2009). The case of oncolytic viruses versus the immune system: waiting on the judgment of Solomon. Hum. Gene Ther. 20, 1119-1132.

Puhlmann, M., Brown, C.K., Gnant, M., et al. (2000). Vaccinia as a vector for tumor-directed gene therapy: biodistribution of a thymidine kinase-deleted mutant. Cancer Gene Ther. 7, 66-73.

Sabatino, M., Zhao, Y., Voiculescu, S., et al. (2008). Conservation of genetic alterations in recurrent melanoma supports the melanoma stem cell hypothesis. Cancer Res. 68, 122-131.

Shida, H., Hinuma, Y., Hatanaka, M., et al. (1988). Effects and virulences of recombinant vaccinia viruses derived from attenuated strains that express the human T-cell leukemia virus type I envelope gene. J. Virol. 62, 4474-4480.

Simon, R., Lam, A., Li, M.C., et al. (2007). Analysis of gene expression data using BRB-ArrayTools. Cancer Inform. 3, 11-17.

Smith, G.L., and Moss, B. (1983). Infectious poxvirus vectors have capacity for at least 25,000 base pairs of foreign DNA. Gene 25, 21-28.

Tysome, J.R., Lemoine, N.R., and Wang, Y. (2009). Combination of anti-angiogenic therapy and virotherapy: arming oncolytic 
viruses with anti-angiogenic genes. Curr. Opin. Mol. Ther. 11, 664-669.

Van Vliet, K., Mohamed, M.R., Zhang, L., et al. (2009). Poxvirus proteomics and virus-host protein interactions. Microbiol. Mol. Biol. Rev. 73, 730-749.

Wang, E., Miller, L.D., Ohnmacht, G.A., et al. (2000). Highfidelity mRNA amplification for gene profiling. Nat. Biotechnol. 18, 457-459.

Wang, E., Voiculescu, S., Le Poole, I.C., et al. (2006). Clonal persistence and evolution during a decade of recurrent melanoma. J. Invest. Dermatol. 126, 1372-1377.

Weibel, S., Raab, V., Yu, Y.A., et al. (2011). Viral-mediated oncolysis is the most critical factor in the late-phase of the tumor regression process upon vaccinia virus infection. BMC Cancer 11, 68.

Willers, I.M., and Cuezva, J.M. (2011). Post-transcriptional regulation of the mitochondrial $\mathrm{H}(+)$-ATP synthase: a key regulator of the metabolic phenotype in cancer. Biochim. Biophys. Acta. 1807, 543-551.

Wong, H.H., Lemoine, N.R., and Wang, Y. (2010). Oncolytic viruses for cancer therapy: overcoming the obstacles. Viruses $2,78-106$.

Worschech, A., Kmieciak, M., Knutson, K.L., et al. (2008). Signatures associated with rejection or recurrence in HER-2/ neu-positive mammary tumors. Cancer Res. 68, 2436-2446.

Worschech, A., Chen, N., Yu, Y.A., et al. (2009). Systemic treatment of xenografts with vaccinia virus GLV-1h68 reveals the immunologic facet of oncolytic therapy. BMC Genomics 10, 301.

Wright, C.F., Oswald, B.W., and Dellis, S. (2001). Vaccinia virus late transcription is activated in vitro by cellular heterogeneous nuclear ribonucleoproteins. J. Biol. Chem. 276, 4068040686.

Yu, Y.A., Galanis, C., Woo, Y., et al. (2009a). Regression of human pancreatic tumor xenografts in mice after a single systemic injection of recombinant vaccinia virus GLV-1h68. Mol. Cancer Ther. 8, 141-151.
Yu, Z., Li, S., Brader, P., et al. (2009b). Oncolytic vaccinia therapy of squamous cell carcinoma. Mol. Cancer 8, 45.

Zaitseva, M., Vollenhoven, B.J., and Rogers, P.A. (2006). In vitro culture significantly alters gene expression profiles and reduces differences between myometrial and fibroid smooth muscle cells. Mol. Hum. Reprod. 12, 187-207.

Zeh, H.J., and Bartlett, D.L. (2002). Development of a replicationselective, oncolytic poxvirus for the treatment of human cancers. Cancer Gene Ther. 9, 1001-1012.

Zhang, Q., Yu, Y.A., Wang, E., et al. (2007). Eradication of solid human breast tumors in nude mice with an intravenously injected light-emitting oncolytic vaccinia virus. Cancer Res. 67, 10038-10046.

Zhang, Q., Liang, C., Yu, Y.A., et al. (2009). The highly attenuated oncolytic recombinant vaccinia virus GLV-1h68: comparative genomic features and the contribution of F14.5L inactivation. Mol. Genet. Genomics 282, 417-435.

Zhu, M., Moore, T., and Broyles, S.S. (1998). A cellular protein binds vaccinia virus late promoters and activates transcription in vitro. J. Virol. 72, 3893-3899.

\author{
Address correspondence to: \\ Dr. Aladar A. Szalay \\ Genelux Corporation \\ San Diego Science Center \\ 3030 Bunker Hill Street, Suite 310 \\ San Diego, CA 92109 \\ E-mail: aaszalay@genelux.com
}

Received for publication March 13, 2012;

accepted after revision August 27, 2012.

Published online: September 11, 2012. 